\title{
'Catastrophic' Thrombosis in a Young Patient With Acute Myeloid Leukemia Presenting Early in the COVID-19 Pandemic - A Case Report
}

\author{
SARAH MATARASSO GREENFELD and TAMAR TADMOR \\ Hematology Unit, B'nai Zion Medical Center, Haifa, Israel
}

\begin{abstract}
Background/Aim: We present the case of a 19year-old male patient diagnosed concomitantly with extensive thromboses (including two intra-cardiac masses and Budd-Chiari syndrome), as well as acute myeloid leukemia. This necessitated prompt deployment of a monitoring and treatment strategy which included twicedaily blood count assessment, multiple platelet transfusions and anti-coagulation therapy with dose-adjustment per blood count during both induction and consolidation chemotherapy. Multiple factors are believed to contribute to the development of thrombosis in acute leukemia such as diffuse intravascular coagulation, cytokine release and chemotherapy. Case Report: Our patient presented early on in the COVID-19 pandemic, delaying his seeking out medical treatment and we suspect this to have contributed to his 'catastrophic' thrombotic presentation. Well-structured guidelines to help clinicians manage these patients are lacking, and most data are from retrospective analyses or case reports. Our patient continued full-dose anticoagulant therapy until successfully undergoing allogeneic stem cell transplant. The thrombi eventually diminished in size, and the patient was not diagnosed with any further thrombotic events. Conclusion: Our case highlights the feasibility of intensive monitoring and provision of platelet transfusion as necessary in order to safely administer low molecular weight heparin from the outset of chemotherapy.
\end{abstract}

Acute myeloid leukemia (AML) is a malignant disorder, characterized by clonal proliferation of immature

This article is freely accessible online.

Correspondence to: Dr. Sarah Matarasso Greenfeld, B'nai Zion Medical Center, 47 Eliyahu Golomb Ave., Haifa 3339419, Israel. Tel: +972 524562511, e-mail: sarah.greenfeld@b-zion.org.il

Key Words: Acute leukemia, thrombosis, anticoagulation, SARSCoV2-Virus, catastrophic thrombosis. hematopoietic stem cells or progenitor cells in the bone marrow. Patients often present with signs and symptoms of bone marrow failure such as infection, bleeding, and shortness of breath. Rarely, thrombosis may be a presenting feature. Here, we present the case of a young patient who presented with classical features of AML along with multiple thromboses including intracardiac masses and thrombosis of inferior vena cava (IVC), pulmonary embolism, and thrombosis of two hepatic veins, as well as a splenic infarct. Treatment included induction therapy for AML, and a personal approach on a daily basis in terms of anticoagulation therapy. The concern and uncertainty in April 2020 regarding the new SARS-CoV-2 virus may have caused a delay in this patient seeking medical evaluation and have been a contributing factor to this exceptional presentation. A short review of the relevant literature is presented along with the management of this precarious and challenging clinical situation.

\section{Case Report}

The patient gave their informed consent to use of their data for this case report.

A 19-year-old previously healthy male presented to the Emergency Department of our hospital during the first wave of the COVID-19 pandemic in April of 2020, with a 2-month history of night sweats, a 6-kg weight loss and dry cough for which he had been prescribed a course of antibiotics. His referral was prompted by the acute onset of severe left upper quadrant abdominal pain and fever. Upon presentation, he was tachycardic (heart rate of 136 beats per min), had a temperature of $39^{\circ} \mathrm{C}$, blood pressure of $136 / 67 \mathrm{mmHg}$ and $100 \%$ oxygen saturation while breathing ambient air. Physical examination was significant for purpura on the upper and lower extremities, hepatosplenomegaly and exquisite abdominal tenderness upon palpation of the left upper quadrant.

Initial laboratory evaluation revealed leukocytosis of $99,000 \times 10^{3} / \mathrm{mm}^{3}$ white blood cells, thrombocytopenia of $18,000 \times 10^{3} / \mathrm{mm}^{3}$ and a hemoglobin level of $5.3 \mathrm{~g} / \mathrm{dl}$. 

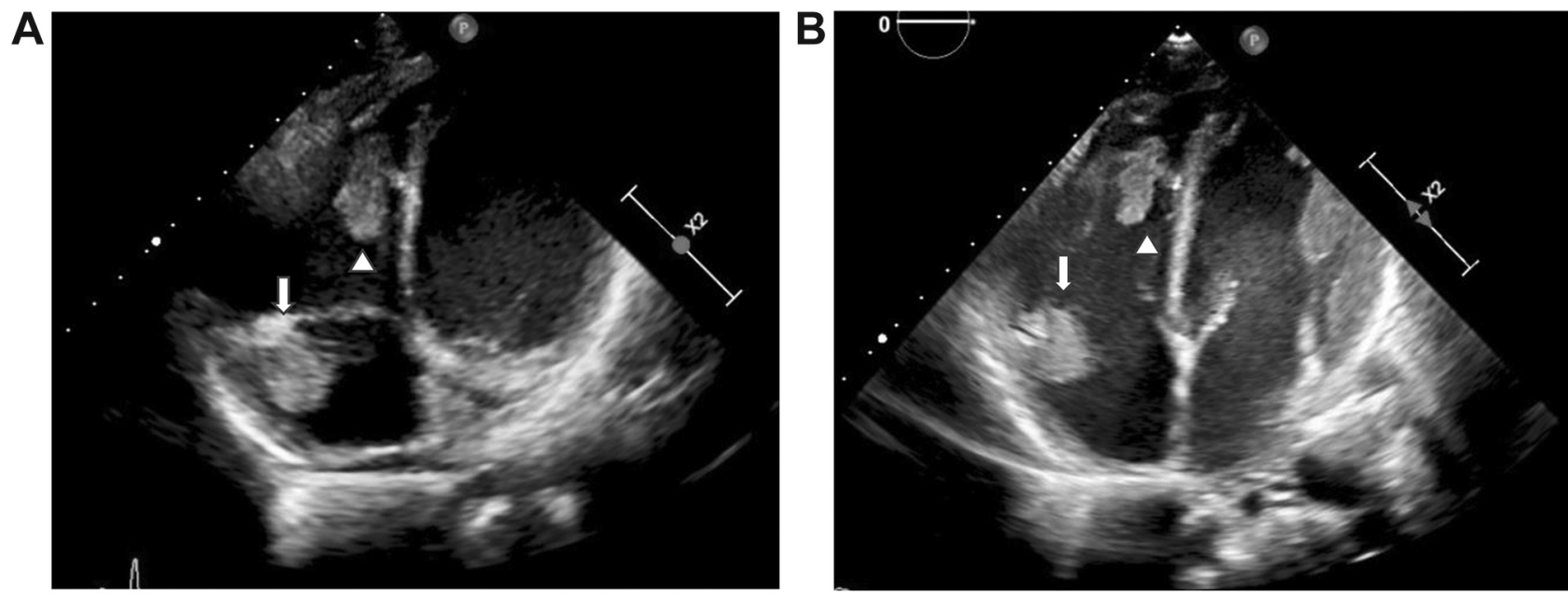

Figure 1. Cardiac masses at diagnosis and following chemotherapy. A: Masses in the right atrium $(1.48 \times 3.11 \mathrm{~cm}$, arrow) and right ventricle $(2.5 \times 2$ $\mathrm{cm}$, arrowhead) on the $4^{\text {th }}$ day of hospitalization. B: Masses decreasing in size following induction and consolidation therapies: right atrium 1.4 1.9 $\mathrm{cm}$ (arrow) and right ventricle $1 \times 2.39 \mathrm{~cm}$.

Coagulation studies showed a prolonged international normalized ratio of 1.73 with a normal prothrombin time, reduced fibrinogen $(115 \mathrm{mg} / \mathrm{dl})$ and an extremely elevated Ddimer level $(21,900 \mathrm{ng} / \mathrm{ml})$. The chemistry panel was significant for elevation of hepatocellular enzymes (aspartate transaminase of $398 \mathrm{U} / 1$ ). A COVID-19 polymerase chain reaction test was negative. Due to the patient's young age and hepatosplenomegaly, the initial working diagnosis was acute lymphoblastic leukemia. However, a blood smear was suggestive of acute myeloid leukemia (AML) and diagnosis was confirmed by flow cytometric analysis of the peripheral blood showing $290 \%$ CD45+ blast forms with strong expression of human leukocyte antigen-D related (HLA-DR), CD34, CD38, CD7 and CD2. Molecular testing revealed an FMS-like tyrosine kinase 3-internal tandem duplication (FLT3-ITD) mutation (allelic ratio $=0.75$ ), and was negative for nucleophosmin-1 (NPM1), and promyelocytic leukemia/retinoic acid receptor alpha $(P M L-R A R A)$ mutation. Karyotype was normal and fluorescence in situ hybridization did not detect translocation $(15 ; 17)$. A non-specific opacity in the lower lobe of the right lung was seen on chest x-ray and an abdominal ultrasound showed hepatosplenomegaly, and thrombi in the hepatic veins and in the inferior vena cava (IVC).

A routine echocardiogram performed prior to the initiation of induction chemotherapy revealed two cardiac masses, in the right atrium and ventricle (Figure 1A). The troponin level was normal and that of NT-pro-brain natriuretic peptide was mildly elevated (434 pg/ml, upper limit of normal=300 $\mathrm{pg} / \mathrm{ml})$. Chest and abdominal computed tomographic scans were ordered, and the patient was also diagnosed with a pulmonary embolism in a right lobar artery (accompanied by a right-sided pleural effusion), thrombosis of the IVC and

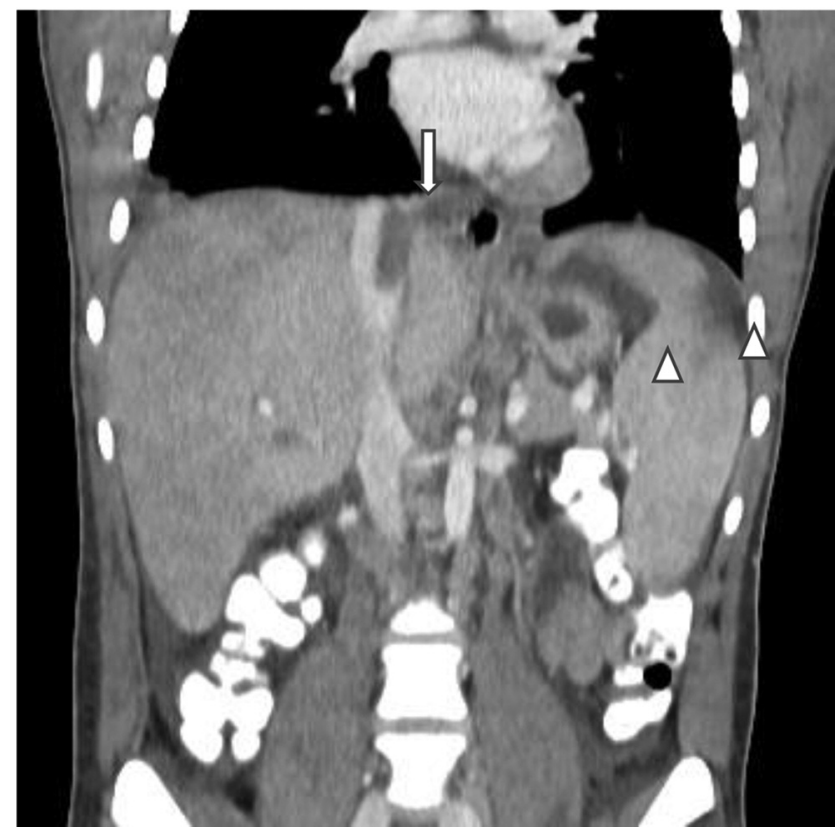

Figure 2. Inferior vena cava thrombosis and splenic infarcts. Filling defect in the inferior vena cava (arrow), and two splenic infarcts (arrowheads).

thrombosis of two hepatic veins. The liver's consistency was heterogeneous and splenic infarcts were detected (Figure 2). Doppler ultrasound of the lower extremities ruled out deep vein thrombosis. Ophthalmological examination revealed leukemic retinopathy and retinal hemorrhage.

A workup for underlying causes of hypercoagulability included testing for lupus anticoagulant and anti-cardiolipin 


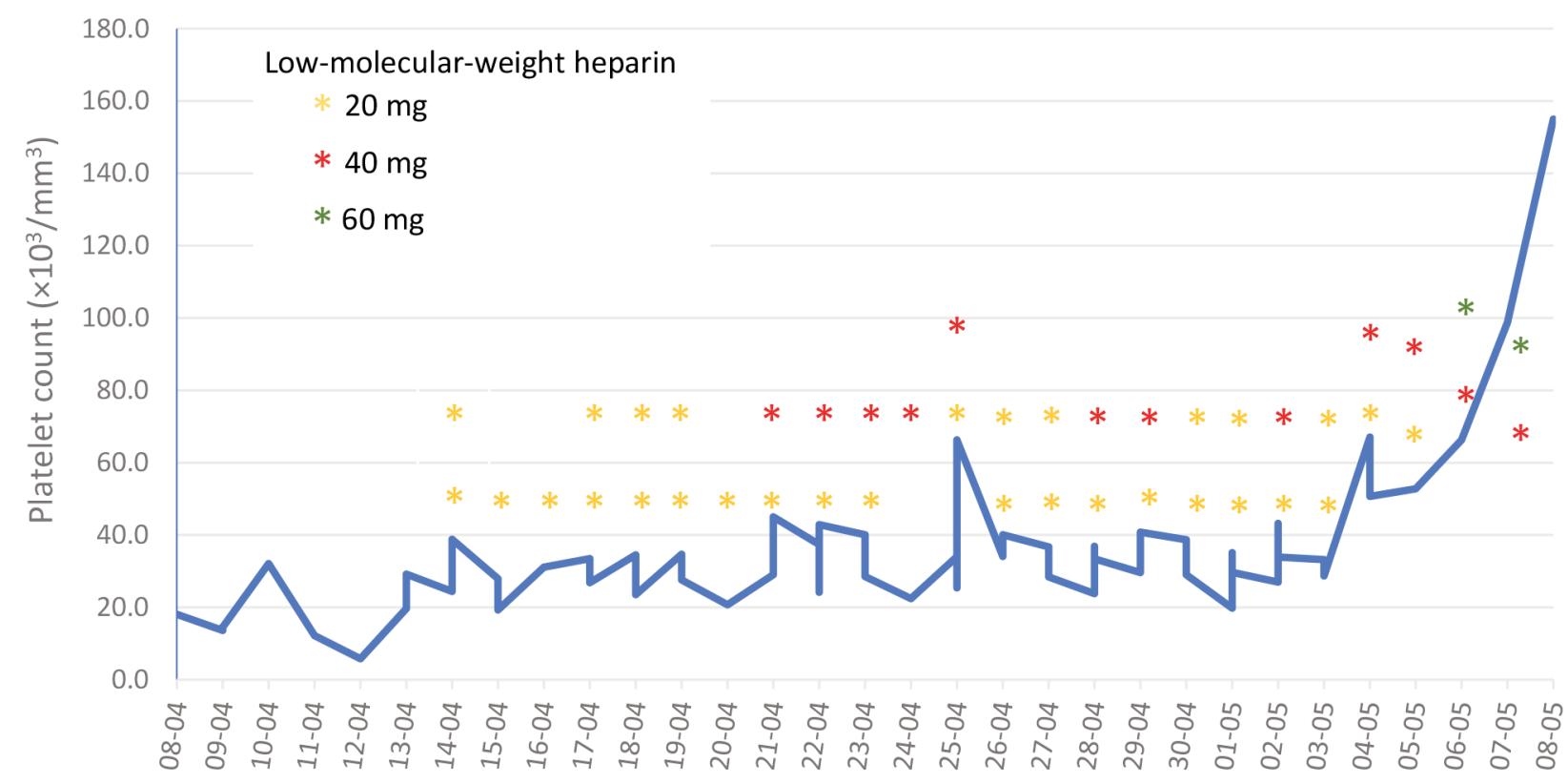

Date in 2020

Figure 3. Dosing of low-molecular-weight heparin during induction chemotherapy according to the platelet count.

$\mathrm{IgM}$ and $\mathrm{IgG}$, as well as anti-B2-glycoprotein $\operatorname{IgM}$ levels, which were within the normal range. Testing for Janus kinase 2 mutation, factor 5 Leiden and prothrombin G20210A mutations, and for paroxysmal nocturnal hematuria was negative. Laboratory testing performed on samples drawn on the sixth day of hospitalization showed low levels of free protein $\mathrm{S}$, and low protein $\mathrm{C}$ and antithrombin 3 activity; the latter two returned to normal range following consolidation therapy, 3 months after initial testing, while the protein $\mathrm{S}$ level remained below normal $(60.5 \%$, lower limit of normal $=70 \%)$ but higher than at diagnosis.

An emergency multi-disciplinary meeting was convened and, despite the complexity of the clinical presentation, it was decided that no invasive diagnostic or therapeutic procedures were to be performed. The etiology of the cardiac masses was uncertain and there was speculation regarding whether these were myeloid chloromas or thrombi.

Induction chemotherapy with cytarabine and daunorubicin was initiated with the addition of midaustarin per protocol. Management also included twice- or thricedaily monitoring of the platelet count and platelet transfusions with mainly single donor platelet as necessary to maintain the platelet count above $30,000 \times 10^{3} / \mathrm{mm}^{3}$ in order to anti-coagulate with low-molecular-weight heparin. Our patient received a total of 14 single donor platelet and 18 random platelet transfusions over the course of his induction therapy. He received either a low, intermediate or therapeutic dose of low-molecular-weight heparin according to the platelet count, with doses ranging from $20 \mathrm{mg}$ once daily to $60 \mathrm{mg}$ twice daily upon bone marrow recovery at the end of induction therapy (Figure 3). No signs of bleeding were recorded. Serial assessment of cardiac troponin and pro-brain natriuretic peptide levels, as well as liver enzymes, was performed during the early treatment period. The troponin level remained normal, while the proBNP level continued to increase to approximately four times above the reference range before normalizing. Hepatocellular enzyme levels were elevated more than 10 times the upper reference range during the first days of hospitalization but had nearly normalized approximately 46 days following admission. Close ultrasonographic, echocardiographic and ophthalmologic follow-up was performed throughout induction and consolidation therapy, and cardiac and hepatic vein thrombi decreased in size over time (Figure 1B). The patient achieved a complete remission following first induction chemotherapy and received consolidation chemotherapy where the same management of anti-coagulation was successfully employed (anti-factor 10A was monitored to ensure appropriate dosing as well as patient compliance). Following a second consolidation therapy, the patient underwent allogeneic bone marrow transplant which was successfully completed; at 4 months following transplant, the patient is now is doing well and continues anticoagulant treatment with once-daily dosing of low-molecular-weight heparin. 


\section{Discussion}

The relationship between malignancy and thrombosis is wellrecognized, both for solid tumors and hematological malignancies (1). Here we present the case of a young patient who presented with AML and multiple thromboses of both arterial and venous types, including two intracardiac thrombi, IVC thrombosis, pulmonary embolus, hepatic vein thrombosis and splenic infarcts. A review of the literature regarding this type of unusual presentation revealed an Italian study by de Stefano et al., who reported the incidence of first symptomatic thrombosis among 24 (6.3\%) out of 379 patients with acute leukemia (both acute lymphoblastic leukemia and AML. Notably, thrombosis was a clinical feature present in $3.2 \%$ of patients with AML (non-acute promyelocytic type) at diagnosis of the 379 patients analyzed (2). Libourel et al. recorded a prevalence of $8.7 \%$ and $10.5 \%$ in cohorts of younger ( $<60$ years) and elderly ( $>60$ years) patients with AML (3).

When a patient is diagnosed with both acute leukemia as well as (venous) thrombosis concomitantly, as was the case with our patient, the therapeutic dilemma regarding anticoagulant management throughout intensive induction chemotherapy (with the inevitable period of severe thrombocytopenia) is at the forefront, and is complicated by the lack of prospective data to help formulate sound guidelines with regards to safe and effective management.

Proposed pathophysiological mechanisms, as well as treatment-associated factors leading to increased thrombotic risk in acute leukemia, can be divided into three broad categories (4): Tumor-related, therapy-related and patient predisposition. Tumor-related mechanisms may include an abundance of tissue factor, cytokines and cancer procoagulant secreted by tumor blasts, tumor-induced activation of platelets, as well as hyperleukocytosis, all of which favor the activation of both platelet and coagulation cascades. Treatment-associated factors include indwelling catheters, chemotherapy, treatment with high-dose steroids and infection (4).

To date, there are few clinical and laboratory parameters that have been shown to improve our ability to identify those patients at highest risk for thrombotic complications, and a widely-validated and useful clinical predictive rule is currently an unmet need. The Khorana risk score, a model developed to stratify thrombotic risk in patients with solid tumors, did not accurately predict thrombosis in patients with AML using clinical and laboratory values at diagnosis in a retrospective cohort of 867 patients, perhaps because of the large number of patients presenting with pancytopenia (5). Recently, a clinical predictive rule for development of venous thromboembolism in patients with acute leukemia was proposed based on three statistically significant variables: Personal history of venous thromboembolism, acute lymphoblastic leukemia and platelet count $>50,000 \times 10^{3} / \mathrm{mm}^{3}$ at baseline); the model was internally validated in a single institution but awaits more extensive validation (6). Diffuse intravascular coagulation at diagnosis and especially an elevated D-dimer level have also been found to be strong predictors for subsequent thrombosis during AML induction therapy (3).

Our patient presented with 'catastrophic' thrombosis at diagnosis of AML, including intracardiac and intrahepatic thrombosis. While the possibility of intracardiac deposition of leukemia cells was considered, in light of the widespread thrombosis, elevated D-dimer level and low fibrinogen, protein C, S and AT3 levels, the clinical picture seems to be consistent with diffuse intravascular coagulation as the main predisposing factor. This, together with the widespread thrombosis, motivated frequent monitoring of the platelet count and multiple platelet transfusions throughout induction and consolidation therapy in order to provide twice-daily anticoagulation when possible.

Perhaps a contributing factor to this exceptional and 'catastrophic' presentation was the fact that the patient's symptoms developed during the beginning of the first wave of the COVID-19 pandemic, which may have delayed his pursuit of medical evaluation and care. However, as this short review of the literature shows, thrombotic complications associated with acute leukemia are not as rare as previously believed.

Our case highlights the feasibility of intensive laboratory monitoring and platelet transfusion support in order to provide anticoagulant treatment even during the induction phase of AML, when the patient is severely thrombocytopenic, and to prevent additional thrombotic complications.

\section{Conflicts of Interest}

The Authors declare they have no conflicts of interest.

\section{Authors' Contributions}

SMG and TT were involved in patient care and management. SMG composed and TT critically reviewed the article.

\section{References}

1 Levitan N, Dowlati A, Remick SC, Tahsildar HI, Sivinski LD, Beyth $\mathrm{R}$ and Rimm AA: Rates of initial and recurrent thromboembolic disease among patients with malignancy versus those without malignancy. Risk analysis using Medicare claims data. Medicine (Baltimore) 78(5): 285-291, 1999. PMID: 10499070. DOI: 10.1097/00005792-19990900000001

2 De Stefano V, Sorà F, Rossi E, Chiusolo P, Laurenti L, Fianchi L, Zini G, Pagano L, Sica S and Leone G: The risk of thrombosis in patients with acute leukemia: occurrence of thrombosis at diagnosis and during treatment. J Thromb 
Haemost 3(9): 1985-1992, 2005. PMID: 16102104. DOI: 10.1111/j.1538-7836.2005.01467.x

3 Libourel EJ, Klerk CPW, van Norden Y, de Maat MPM, Kruip MJ, Sonneveld P, Löwenberg B and Leebeek FWG: Disseminated intravascular coagulation at diagnosis is a strong predictor for thrombosis in acute myeloid leukemia. Blood 128(14): 1854-1861, 2016. PMID: 27354723. DOI: 10.1182/ blood-2016-02-701094

4 Del Principe MI, Del Principe D and Venditti A: Thrombosis in adult patients with acute leukemia. Curr Opin Oncol 29(6): 448454, 2017. PMID: 28841588. DOI: $10.1097 / \mathrm{CCO} .00000000$ 00000402

5 Mirza AS, Yun S, Ali NA, Shin H, O’Neil JL, Elharake M, Schwartz D, Robinson K, Nowell E, Engle G, Badat I, Brimer T, Kuc A, Sequeira A, Mirza S, Sikaria D, Vera JD, Hackney N,
Abusrur S, Jesurajan J, Kuang J, Patel S, Khalil S, Bhaskar S, Beard A, Abuelenen T, Ratnasamy K, Visweshwar N, Komrokji $\mathrm{R}$ and Jaglal $\mathrm{M}$ : Validation of the Khorana score in acute myeloid leukemia patients: a single-institution experience. Thromb J 17: 13, 2019. PMID: 31303864. DOI: 10.1186/ s12959-019-0202-z

6 Al-Ani F, Wang YP and Lazo-Langner A: Development of a clinical prediction rule for venous thromboembolism in patients with acute leukemia. Thromb Haemost 120(2): 322-328, 2020. PMID: 31893562. DOI: 10.1055/s-0039-3400303

Received April 30, 2021

Revised June 15, 2021

Accepted June 22, 2021 